\title{
Erratum to "A New Class of Particle Filters for Random Dynamic Systems with Unknown Statistics"
}

\author{
Joaquín Míguez, ${ }^{1}$ Mónica F. Bugallo, ${ }^{2}$ and Petar M. Djurićc ${ }^{2}$ \\ ${ }^{1}$ Departamento de Teoría de la Señal y las Comunicaciones, Universidad Carlos III de Madrid, 28911 Leganes, Spain \\ ${ }^{2}$ Department of Electrical and Computer Engineering, Stony Brook University, Stony Brook, NY 11794, USA
}

Received 28 August 2005; Accepted 9 November 2005

Recommended for Publication by Marc Moonen

We have found an error in the proof of Lemma 1 presented in our paper "A New Class of Particle Filters for Random Dynamic Systems with Unknown Statistics" (EURASIP Journal on Applied Signal Processing, 2004). In the sequel, we provide a restatement of the lemma and a corrected (and simpler) proof. We emphasize that the original result in the said paper still holds true. The only difference with the new statement is the relaxation of condition (3), which becomes less restrictive.

Copyright (c) 2006 Hindawi Publishing Corporation. All rights reserved.

Lemma 1 in [1] should be as follows.

Lemma 1. Let $\left\{\mathbf{x}_{t}^{(i)}\right\}_{i=1}^{M}$ be a set of particles drawn at time $t$ using the propagation $p d f p_{t}^{M^{\prime}}(\mathbf{x})$, let $\mathbf{y}_{1: t}$ be a fixed bounded sequence of observations, let $\Delta \mathfrak{C}\left(\mathbf{x} \mid \mathbf{y}_{t}\right) \geq 0$ be a continuous cost function, bounded in $S\left\{\mathbf{x}_{t}^{\mathrm{opt}}, \varepsilon\right\}$, with a minimum at $\mathbf{x}=$ $\mathbf{x}_{t}^{\text {opt }}$, and let $\mu_{t}: A \subseteq\left\{\mathbf{x}_{t}^{(i)}\right\}_{i=1}^{M} \rightarrow[0, \infty)$ be a set function defined as

$$
\mu_{t}\left(A \subseteq\left\{\mathbf{x}_{t}^{(i)}\right\}_{i=1}^{M}\right)=\sum_{\mathbf{x} \in A} \mu\left(\Delta \mathcal{C}\left(\mathbf{x} \mid \mathbf{y}_{t}\right)\right) .
$$

If the following three conditions are met:

(1) Any ball with center at $\mathbf{x}_{t}^{\text {opt }}$ has a nonzero probability under the propagation density, that is,

$$
\int_{S\left\{\mathbf{x}_{t}^{\mathrm{opt}}, \varepsilon\right\}} p_{t}^{M^{\prime}}(\mathbf{x}) d \mathbf{x}=\gamma>0, \quad \forall \varepsilon>0,
$$

(2) the supremum of the function $\mu(\Delta \mathfrak{C}(\cdot \mid \cdot))$ for points outside $S\left(\mathbf{x}_{t}^{\text {opt }}, \varepsilon\right)$ is a finite constant, that is,

$$
\mathbf{S}_{\text {out }}=\sup _{\mathbf{x}_{t} \in \mathbb{R}^{L x} \backslash S\left(\mathbf{x}_{t}^{\text {opt }}, \varepsilon\right)}\left\{\mu\left(\Delta \mathcal{C}\left(\mathbf{x}_{t} \mid \mathbf{y}_{t}\right)\right)\right\}<\infty
$$

(3) the expected value of $1 / \mu_{t}\left(\left\{\mathbf{x}_{t}^{(i)}\right\}_{i=1}^{M}\right)$ satisfies

$$
\lim _{M \rightarrow \infty} E\left(\frac{1}{\mu_{t}\left(\left\{\mathbf{x}_{t}^{(i)}\right\}_{i=1}^{M}\right) / M}\right)=0,
$$

then

$$
\lim _{M \rightarrow \infty} \operatorname{Pr}\left[1-\frac{\mu_{t}\left(S^{M}\left(\mathbf{x}_{t}^{\mathrm{opt}}, \varepsilon\right)\right)}{\mu_{t}\left(\left\{\mathbf{x}_{t}^{(i)}\right\}_{i=1}^{M}\right)} \geq \delta\right]=0, \quad \forall \delta>0,
$$

where $\operatorname{Pr}[\cdot]$ denotes probability, that is,

$$
\left.\lim _{M \rightarrow \infty} \frac{\mu_{t}\left(S^{M}\left(\mathbf{x}_{t}^{\text {opt }}, \varepsilon\right)\right)}{\mu_{t}\left(\left\{\mathbf{x}_{t}^{(i)}\right\}_{i=1}^{M}\right)}=1 \quad \text { (i.p. }\right),
$$

where i.p. stands for "in probability."

Proof. The proof is based on Markov inequality. We write

$$
\begin{aligned}
\lim _{M \rightarrow \infty} \operatorname{Pr} & {\left[1-\frac{\mu_{t}\left(S^{M}\left(\mathbf{x}_{t}^{\mathrm{opt}}, \varepsilon\right)\right)}{\mu_{t}\left(\left\{\mathbf{x}_{t}^{(i)}\right\}_{i=1}^{M}\right)} \geq \delta\right] } \\
= & \lim _{M \rightarrow \infty} \operatorname{Pr}\left[\frac{\mu_{t}\left(\left\{\mathbf{x}_{t}^{(i)}\right\}_{i=1}^{M}\right)-\mu_{t}\left(S^{M}\left(\mathbf{x}_{t}^{\mathrm{opt}}, \varepsilon\right)\right)}{\mu_{t}\left(\left\{\mathbf{x}_{t}^{(i)}\right\}_{i=1}^{M}\right)} \geq \delta\right] \\
= & \lim _{M \rightarrow \infty} \operatorname{Pr}\left[\frac{\mu_{t}\left(\left\{\mathbf{x}_{t}^{(i)}\right\}_{i=1}^{M} \backslash S^{M}\left(\mathbf{x}_{t}^{\mathrm{opt}}, \varepsilon\right)\right)}{\mu_{t}\left(\left\{\mathbf{x}_{t}^{(i)}\right\}_{i=1}^{M}\right)} \geq \delta\right] .
\end{aligned}
$$

Using the second condition, we infer that

$$
\begin{gathered}
\lim _{M \rightarrow \infty} \operatorname{Pr}\left[\frac{\mu_{t}\left(\left\{\mathbf{x}_{t}^{(i)}\right\}_{i=1}^{M} \backslash S^{M}\left(\mathbf{x}_{t}^{\text {opt }}, \varepsilon\right)\right)}{\mu_{t}\left(\left\{\mathbf{x}_{t}^{(i)}\right\}_{i=1}^{M}\right)} \geq \delta\right] \\
\quad \leq \lim _{M \rightarrow \infty} \operatorname{Pr}\left[\frac{M S_{\text {out }}}{\mu_{t}\left(\left\{\mathbf{x}_{t}^{(i)}\right\}_{i=1}^{M}\right)} \geq \delta\right] .
\end{gathered}
$$


Finally, we apply Markov inequality to the last expression on the right and obtain

$$
\begin{array}{r}
\lim _{M \rightarrow \infty} \operatorname{Pr}\left[\frac{\mu_{t}\left(\left\{\mathbf{x}_{t}^{(i)}\right\}_{i=1}^{M} \backslash S^{M}\left(\mathbf{x}_{t}^{\text {opt }}, \varepsilon\right)\right)}{\mu_{t}\left(\left\{\mathbf{x}_{t}^{(i)}\right\}_{i=1}^{M}\right)} \geq \delta\right] \\
\leq \frac{S_{\text {out }}}{\delta} \lim _{M \rightarrow \infty}\left[E\left(\frac{1}{\mu_{t}\left(\left\{\mathbf{x}_{t}^{(i)}\right\}_{i=1}^{M}\right) / M}\right)\right] .
\end{array}
$$

Clearly, if

$$
\lim _{M \rightarrow \infty}\left[E\left(\frac{1}{\mu_{t}\left(\left\{\mathbf{x}_{t}^{(i)}\right\}_{i=1}^{M}\right) / M}\right)\right]=0
$$

we can claim that

$$
\left.\lim _{M \rightarrow \infty} \frac{\mu_{t}\left(S^{M}\left(\mathbf{x}_{t}^{\mathrm{opt}}, \varepsilon\right)\right)}{\mu_{t}\left(\left\{\mathbf{x}_{t}^{(i)}\right\}_{i=1}^{M}\right)}=1 \quad \text { (i.p. }\right)
$$

\section{REFERENCES}

[1] J. Míguez, M. F. Bugallo, and P. M. Djurić, "A new class of particle filters for random dynamic systems with unknown statistics," EURASIP Journal on Applied Signal Processing, vol. 2004, no. 15, pp. 2278-2294, 2004.

Joaquín Míguez was born in Ferrol, Galicia, Spain, in 1974. He obtained the Licenciado en Informatica (M.S.) and Doctor en Informatica (Ph.D.) degrees from Universidade da Coruña, Spain, in 1997 and 2000, respectively. Late in 2000, he joined Departamento de Electrónica e Sistemas, Universidade da Coruña, where he became an Associate Professor in July 2003. His research interests are in the field of statistical signal processing, with emphasis on the topics of Bayesian analysis, sequential Monte Carlo methods, adaptive filtering, stochastic optimization, and their applications to multiuser communications, smart antenna systems, target tracking, and vehicle positioning and navigation.

Mónica F. Bugallo received the Ph.D. degree in computer engineering from the University of A Coruña, Spain, in 2001. From 1998 to 2000 she was with the Departamento de Electrónica y Sistemas at the Universidade da Coruña, Spain, where she worked in interference cancellation applied to multiuser communication systems. In 2001, she joined the Department of Electrical and Computer Engineering at Stony

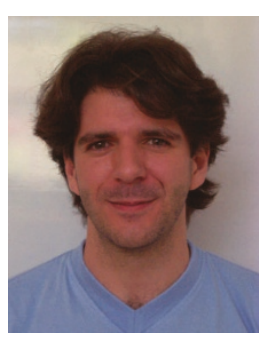

Brook University, where she is currently an Assistant Professor and teaches courses in digital communications and information theory. Her research interests lie in the area of statistical signal processing and its applications to different disciplines including communications and biology.

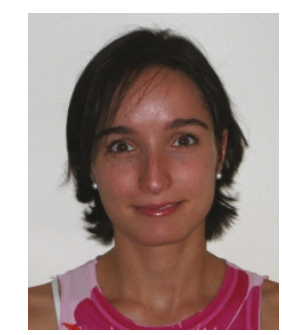

Petar M. Djurić received his B.S. and M.S. degrees in electrical engineering from the University of Belgrade, in 1981 and 1986, respectively, and his $\mathrm{Ph} . \mathrm{D}$. degree in electrical engineering from the University of Rhode Island, in 1990. From 1981 to 1986 he was Research Associate with the Institute of Nuclear Sciences, Vinca, Belgrade. Since 1990 he has been with Stony Brook University, where he is Professor a in the Depart-

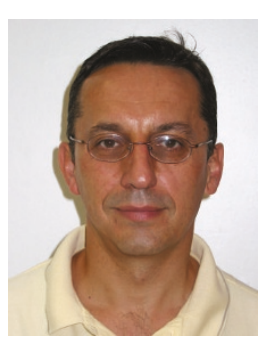
ment of Electrical and Computer Engineering. He works in the area of statistical signal processing, and his primary interests are in the theory of modeling, detection, estimation, and time series analysis, and its application to a wide variety of disciplines including wireless communications and biomedicine. 